\title{
ON THE COMPLEX OSCILLATION OF HIGHER ORDER LINEAR DIFFERENTIAL EQUATIONS
}

\author{
KAi LiU AND LiAn-ZhONG YANG
}

\begin{abstract}
In this paper, we investigate the growth of solutions and the existence of subnormal solutions for a class of higher order linear differential equations. We obtain some results which improve and extend the results of Chen-Shon [2] and Gundersen-Steinbart [5].
\end{abstract}

\section{Introduction}

In the study of the oscillation theory of complex differential equations, the growth of solutions is a very important property. For linear differential equations of the form

$$
f^{(n)}+a_{n-1}(z) f^{(n-1)}+\cdots+a_{0}(z) f=0,
$$

where $a_{0}(z), \ldots, a_{n-1}(z)$ are polynomials, it is known that every entire solution of equation (1) must be of finite order, and if some of the coefficients $a_{j}(z)(0 \leq$ $j \leq n-1)$ are replaced by transcendental entire functions, then equation (1) has at least one solution of infinite order. This can be proved by mainly using the Wiman-Valiron theory $[6,8]$.

In this paper, we assume that the reader is familiar with the fundamental results and the standard notation of the value distribution theory of meromorphic functions $[8,10]$. We denote the order of growth of $f$ by $\sigma(f)$. In addition, let us define inductively, for $r \in[0,+\infty)$, $\exp ^{[1]} r=e^{r}$ and $\exp ^{[n+1]} r=$ $\exp \left(\exp ^{[n]} r\right), n \in \mathbb{N}$. For all $r$ sufficiently large, define $\log ^{[1]} r=\log r$ and $\log ^{[n+1]} r=\log \left(\log { }^{[n]} r\right), n \in \mathbb{N}$. To express the rate of growth of meromorphic functions of infinite order, we recall the following definitions [7].

Definition 1.1. The iterated $p$-order $\sigma_{p}(f)$ of a meromorphic function $f$ is defined by

$$
\sigma_{p}(f)=\limsup _{r \rightarrow \infty} \frac{\log ^{[p]} T(r, f)}{\log r}, \quad p \in \mathbb{N} .
$$

Received January 23, 2008; Revised July 15, 2008.

2000 Mathematics Subject Classification. 34M10, 30D35.

Key words and phrases. periodic differential equation, subnormal solution, e-type order. The authors were supported by the NNSF of China (NO. 10671109). 
Definition 1.2. Suppose $f \neq 0$ is a solution of equation (1) and if $f$ satisfies the condition

$$
\limsup _{r \rightarrow \infty} \frac{\log T(r, f)}{r}=0
$$

then we say that $f$ is a nontrivial subnormal solution of equation (1).

Chiang and Gao [3] gave the definition of the e-type order of meromorphic function as follows:

Definition 1.3. Let $f$ be a meromorphic function. Define

$$
\sigma_{e}(f)=\limsup _{r \rightarrow \infty} \frac{\log T(r, f)}{r}
$$

to be the e-type order of $f$.

From Definition 1.3, we know that if $\sigma_{e}(f)=0$, then $f$ must be subnormal. The following results are obviously.

(i) If $0<\sigma_{e}(f)<\infty$, then $\sigma_{2}(f)=1$,

(ii) if $\sigma_{2}(f)<1$, then $\sigma_{e}(f)=0$,

(iii) if $\sigma_{2}(f)=\infty$, then $\sigma_{e}(f)=\infty$.

Thus the iterated order and the e-type order can be applied to get a more precise estimate of the growth of meromorphic function with infinite order. Wittich [9] investigated the subnormal solution of the equation

$$
f^{\prime \prime}+P\left(e^{z}\right) f^{\prime}+Q\left(e^{z}\right) f=0,
$$

where $P(z)$ and $Q(z)$ are nonconstant polynomials in $z$.

Theorem A. If $f \neq 0$ is a subnormal solution of (2), then $f$ must have the form

$$
f(z)=e^{c z}\left(h_{0}+h_{1} e^{z}+\cdots+h_{m} e^{m z}\right),
$$

where $m \geq 0$ is an integer, $c, h_{0}, \ldots, h_{m}$ are constants and $h_{0}, h_{m} \neq 0$.

Based on the comparison of degrees of $P$ and $Q$, Gundersen and Steinbart [5] improved Theorem A and obtained the following theorem.

Theorem B. The following statements hold with regard to the subnormal solutions of equation (2),

(i) if $\operatorname{deg} P>\operatorname{deg} Q$ and $Q \neq 0$, then any subnormal solution $f \neq 0$ of equation (2) must have the form

$$
f(z)=\sum_{k=0}^{m} h_{k} e^{-k z},
$$

where $m \geq 1$ is an integer and $h_{0}, \ldots, h_{m}$ are constants, $h_{0}, h_{m} \neq 0$,

(ii) if $\operatorname{deg} P \geq 1$ and $Q=0$, then any subnormal solution of equation (2) must be a constant,

(iii) if $\operatorname{deg} P<\operatorname{deg} Q$, then the subnormal solution of equation (2) is $f=0$. 
Gundersen and Steinbart [5] also considered non-homogeneous differential equations

$$
f^{\prime \prime}+P\left(e^{z}\right) f^{\prime}+Q\left(e^{z}\right) f=R_{1}\left(e^{z}\right)+R_{2}\left(e^{-z}\right),
$$

where $P(z), Q(z), R_{1}(z)$ and $R_{2}(z)$ are polynomials in $z$ and obtained the following theorem.

Theorem C. Suppose $f \neq 0$ is a subnormal solution of (3) and $\operatorname{deg} P>\operatorname{deg} Q$.

(i) If $\operatorname{deg} P>\operatorname{deg} R_{1}$, then $f$ must have the form

$$
f(z)=S_{1}\left(e^{z}\right)+S_{2}\left(e^{-z}\right),
$$

where $S_{1}(z)$ and $S_{2}(z)$ are polynomials in $z$;

(ii) if $\operatorname{deg} P \leq \operatorname{deg} R_{1}$, then $f$ must have the form

$$
f(z)=c z \sum_{k=0}^{m} a_{k} e^{-k z}+S_{1}\left(e^{z}\right)+S_{2}\left(e^{-z}\right),
$$

where $m \geq 0$ is an integer, $c, a_{0}, \ldots, a_{m}$ are constants with $a_{0}, a_{m} \neq 0$, and $S_{1}(z), S_{2}(z)$ are polynomials in $z$.

They also raised the following question in [5]:

Question. Whether equation (3) in Theorem $\mathrm{C}$ can be generalized to equation (4) $f^{\prime \prime}+\left[P_{1}\left(e^{z}\right)+P_{2}\left(e^{-z}\right)\right] f^{\prime}+\left[Q_{1}\left(e^{z}\right)+Q_{2}\left(e^{-z}\right)\right] f=R_{1}\left(e^{z}\right)+R_{2}\left(e^{-z}\right) ?$

With regard to above question, Chen and Shon [2] investigated the existence of subnormal solutions of equation (4) and its corresponding homogeneous equation

$$
f^{\prime \prime}+\left[P_{1}\left(e^{z}\right)+P_{2}\left(e^{-z}\right)\right] f^{\prime}+\left[Q_{1}\left(e^{z}\right)+Q_{2}\left(e^{-z}\right)\right] f=0 .
$$

They obtained some results about the subnormal solutions and the growth of solutions of (4) and (5). Their results can be stated as follows.

Theorem D. Let $P_{j}(z), Q_{j}(z)(j=1,2)$ be polynomials in $z$. If

$$
\operatorname{deg} Q_{1}>\operatorname{deg} P_{1} \quad \text { or } \quad \operatorname{deg} Q_{2}>\operatorname{deg} P_{2} \text {, }
$$

then the differential equation (5) has no nontrivial subnormal solutions, and every nontrivial solution of (5) satisfies $\sigma_{2}(f)=1$.

Theorem E. Let $P_{j}(z), Q_{j}(z)(j=1,2)$ be polynomials in $z$, and $R_{1}+R_{2} \neq 0$. If $P_{j}(z), Q_{j}(z)$ satisfy the conditions (6), then

(i) equation (4) has at most a subnormal solution $f_{0}$, and $f_{0}=S_{1}\left(e^{z}\right)+$ $S_{2}\left(e^{-z}\right)$, where $S_{1}(z)$ and $S_{2}(z)$ are polynomials in $z$;

(ii) all solutions $f$ of equation (4) satisfy $\sigma_{2}(f)=1$ except the subnormal solution in (i).

The main purpose of this paper is to improve Theorems D, E to higher order periodic differential equations. Furthermore, we replace the exponent $z$ or $-z$ 
with nonconstant entire functions to the former theorems. We obtained the following results.

Theorem 1.1. Let $P_{j}(z), Q_{j}(z)(j=0,1, \ldots, n-1)$ be polynomials in $z$. If

$$
\operatorname{deg} P_{0}>\operatorname{deg} P_{j} \quad \text { or } \quad \operatorname{deg} Q_{0}>\operatorname{deg} Q_{j} \quad j=1, \ldots, n-1,
$$

then the $n$-th order differential equation

$$
f^{(n)}+\left[P_{n-1}\left(e^{z}\right)+Q_{n-1}\left(e^{-z}\right)\right] f^{(n-1)}+\cdots+\left[P_{0}\left(e^{z}\right)+Q_{0}\left(e^{-z}\right)\right] f=0
$$

has no nontrivial subnormal solution, and every nontrivial solution satisfies $\sigma_{2}(f)=1$

Remark 1.1. Obviously, equation (7) is more general than equation (5) in Theorem D. The following example shows that, in Theorem 1.1, if there exists $\operatorname{deg} P_{i}=\operatorname{deg} P_{j}$ and $\operatorname{deg} Q_{i}=\operatorname{deg} Q_{j}, i \neq j$, then equation (7) may have a nontrivial subnormal solution.

Example. A subnormal solution $f=e^{-z}$ satisfies the following equation

$$
f^{(n)}+f^{(n-1)}+\cdots+f^{\prime \prime}+\left(e^{2 z}+e^{-2 z}\right) f^{\prime}+\left(e^{2 z}+e^{-2 z}\right) f=0,
$$

where $n$ is an odd number.

Remark 1.2. If $\operatorname{deg} P_{j}>\operatorname{deg} P_{0}$, then equation (7) may have a nontrivial subnormal solution, which can be seen by the following example.

Example. A subnormal solution $f=e^{-z}-1$ satisfies the equation

$$
f^{\prime \prime \prime}+e^{z} f^{\prime \prime}+\left(e^{2 z}-e^{-z}\right) f^{\prime}-\left(e^{z}+e^{-z}\right) f=0 .
$$

Corollary 1.1. Under the assumptions of Theorem 1.1, the following equation

$$
\begin{aligned}
f^{(n)} & +\left[P_{n-1}\left(e^{z}\right)+Q_{n-1}\left(e^{-z}\right)\right] f^{(n-1)}+\cdots+\left[P_{1}\left(e^{z}\right)+Q_{1}\left(e^{-z}\right)\right] f^{\prime} \\
& +\left[P_{0}\left(e^{z}\right)+Q_{0}\left(e^{-z}\right)\right] f=R_{1}\left(e^{z}\right)+R_{2}\left(e^{-z}\right),
\end{aligned}
$$

has at most a subnormal solution $f_{0}$, and other solutions $f$ satisfy $\sigma_{2}(f)=1$.

Theorem 1.2. Let $A(z), B(z)$ be nonconstant entire functions, and let $P(z)$, $Q(z)$ be polynomials in $z$. Then

$$
f^{\prime \prime}+P\left(e^{A}\right) f^{\prime}+Q\left(e^{B}\right) f=0
$$

has no nontrivial subnormal solutions except two cases:

(1) $A(z), B(z)$ are polynomials in $z, \operatorname{deg} A=\operatorname{deg} B$ and $\operatorname{deg} P=\operatorname{deg} Q$.

(2) $A(z), B(z)$ are transcendental entire functions, $\operatorname{deg} P=\operatorname{deg} Q$ and $\sigma_{p}(A(z))=\sigma_{p}(B(z))$.

Remark 1.3. From the conclusion of Theorem A, we easily find that $\operatorname{deg} P=$ $\operatorname{deg} Q$, which can be seen a special case of (1) in Theorem 1.2. 


\section{Some lemmas}

The following two results easily get from the classical Wiman-Valiron theory.

Lemma 2.1. Let $A_{i}(i=0,1, \ldots, n-1)$ be finite order entire functions. If $f$ is a solution of differential equation

$$
f^{(n)}+A_{n-1} f^{(n-1)}+\cdots+A_{0} f=0
$$

then $\sigma_{2}(f) \leq \max \left\{\sigma\left(A_{i}\right)\right\}$.

Lemma 2.2. Let $f(z)$ be an entire function of order $\sigma$, and let $\nu_{f}(r)$ be the central index of $f$. Then

$$
\sigma=\limsup _{r \rightarrow \infty} \frac{\log ^{+} \nu_{f}(r)}{\log r}
$$

Lemma $2.3([4])$. Let $f$ be a transcendental meromorphic function, and let $\alpha>1$ be a given constant. Then there exists a set $E \subset(1,+\infty)$ with finite logarithmic measure and a constant $B>0$ that depends only on $\alpha$ and $m, n(0 \leq$ $m<n)$ such that for all $|z|=r \notin(0,1) \cup E$, we have

$$
\left|\frac{f^{(n)}(z)}{f^{(m)}(z)}\right| \leq\left(\frac{T(\alpha r, f)}{r} \log ^{\alpha} r \log T(\alpha r, f)\right)^{n-m} .
$$

Lemma 2.4. Let $f$ be a transcendental entire function, and let $s$ be a positive integer. Then there exists one sequence $\left\{r_{k}\right\}\left(r_{k} \rightarrow \infty\right)$ such that $\left|\frac{f(z)}{f^{(s)}(z)}\right| \leq r_{k}^{2 s}$ holds for enough large $r_{k}$, as $|z|=r_{k}$.

Proof. Using the similarly method as the proof of Lemma 14 in [1], we get that there exists one sequence $\left\{r_{k}\right\}\left(r_{k} \rightarrow \infty\right)$ such that

$$
\frac{f^{(s)}(z)}{f(z)}=\left(\frac{\nu_{f}\left(r_{k}\right)}{z}\right)^{s}(1+o(1)), \quad n \in \mathbb{N},
$$

holds for enough large $\left\{r_{k}\right\}$, as $|z|=r_{k}$ and $|f(z)|=M\left(r_{k}, f\right)$.

On the other hand, since $f$ is an entire function, from Lemma 2.2, which implies that for any $\varepsilon>0$, we have $\nu_{f}\left(r_{k}\right) \geq r_{k}^{\sigma-\varepsilon}$, where $\sigma-\varepsilon$ can be replaced with a large enough real number $M$ if $\sigma=\infty$. By calculation, we get $\left|\frac{f(z)}{f^{(s)}(z)}\right| \leq$ $r_{k}^{(1-\sigma+\varepsilon) s} \leq r_{k}^{2 s}$

Lemma $2.5([8])$. Let $g:(0,+\infty) \rightarrow R, h:(0,+\infty) \rightarrow R$ be monotone increasing functions such that $g(r) \leq h(r)$ outside of an exceptional set $E$ of finite linear measure. Then, for any $\alpha>1$, there exists $r_{0}>0$ such that $g(r) \leq h(\alpha r)$ for all $r>r_{0}$. 


\section{Proofs of main results}

Proof of Theorem 1.1. Suppose that

$$
\begin{aligned}
& P_{j}(z)=a_{j m_{j}} z^{m_{j}}+a_{j m_{j-1}} z^{m_{j-1}}+\cdots+a_{j 1} z+a_{j 0}, \\
& Q_{j}(z)=b_{j n_{j}} z^{n_{j}}+b_{j n_{j-1}} z^{n_{j-1}}+\cdots+b_{j 1} z+b_{j 0},
\end{aligned}
$$

where $j=0,1, \ldots, n-1$ and $m_{j}, n_{j} \in \mathbb{Z}$.

Let $f \neq 0$ be a solution of equation (7). Then $f$ must be an entire function. From Lemma 2.1, we get

$$
\sigma_{2}(f) \leq \max \left\{\sigma\left(P_{j}\left(e^{z}\right)\right), \sigma\left(Q_{j}\left(e^{z}\right)\right)\right\}=1 .
$$

The following, we will prove $\sigma_{2}(f) \geq 1$. By Lemma 2.3, we see that there exists a subset $E \subset(1,+\infty)$ with finite logarithmic measure and a constant $B>0$ such that for all $z$ satisfying $|z| \notin(0,1) \cup E$, and we have

$$
\left|\frac{f^{(j)}(z)}{f(z)}\right| \leq B[T(2 r, f)]^{j+1}, j=1,2, \ldots, n-1 .
$$

If $\operatorname{deg} P_{0}>\operatorname{deg} P_{j}$, then we take $z=r$. If $\operatorname{deg} Q_{0}>\operatorname{deg} Q_{j}$, we take $z=-r$. Combining (7) with(10), for a sufficiently large $r$ and $r \notin(0,1) \cup E$, we obtain

$$
\begin{aligned}
\left|P_{0}\left(e^{z}\right)+Q_{0}\left(e^{-z}\right)\right|= & \left|a_{0 m_{0}}\right| e^{m_{0} r}(1+o(1)) \\
\leq & \left|\frac{f^{(n)}}{f}\right|+\left|P_{n-1}\left(e^{z}\right)+Q_{n-1}\left(e^{-z}\right)\right|\left|\frac{f^{(n-1)}}{f}\right|+\cdots \\
& +\left|P_{1}\left(e^{z}\right)+Q_{1}\left(e^{-z}\right)\right|\left|\frac{f^{\prime}}{f}\right| \\
\leq & B[T(2 r, f)]^{n+1}+\left|a_{n-1 m_{n-1}}\right| e^{m_{n-1} r} B[T(2 r, f)]^{n}(1+o(1)) \\
& +\cdots+\left|a_{1 m_{1}}\right| e^{m_{1} r} B[T(2 r, f)]^{2}(1+o(1)) \\
\leq & B[T(2 r, f)]^{n+2} M e^{\max \left\{m_{1}, \ldots, m_{n-1}\right\} r}(1+o(1)),
\end{aligned}
$$

where $M=\max \left\{\left|a_{n-1 m_{n-1}}\right|, \ldots,\left|a_{1 m_{1}}\right|\right\}$. Since $m_{0}>\max \left\{m_{1}, \ldots, m_{n-1}\right\}$, utilizing (11) and Lemma 2.5, we get $\sigma_{2}(f) \geq 1$. Thus, $\sigma_{2}(f)=1$.

Now we will prove any solution $f(\neq 0)$ is not subnormal. Otherwise, if $f$ is subnormal, from Definition 1.2, for any given $\varepsilon, 0<\varepsilon<\frac{1}{4 n+8}, f$ satisfies

$$
T(r, f) \leq e^{r \varepsilon} \text {. }
$$

As we take $z=r \notin(0,1) \cup E$, from (11) and (12), we obtain that

$$
\begin{aligned}
\left|a_{0 m_{0}}\right| e^{m_{0} r}(1+o(1)) & \leq B e^{(2 n+4) r \varepsilon} M e^{\max \left\{m_{1}, \ldots, m_{n-1}\right\} r}(1+o(1)) \\
& \leq B e^{\frac{1}{2} r} M e^{\max \left\{m_{1}, \ldots, m_{n-1}\right\} r}(1+o(1)) .
\end{aligned}
$$

Since $m_{0}>\max \left\{m_{1}, \ldots, m_{n-1}\right\}$, we get a contradiction as $r \rightarrow \infty$. Hence, equation (7) has no nontrivial subnormal solution, thus we have completed the proof of Theorem 1.1. 
Proof of Corollary 1.1. If $f_{1}$ and $f_{2}$ are two distinct subnormal solutions of equation (8), so $f_{2}-f_{1}$ is a subnormal solution of the corresponding homogeneous equation (7), which contradicts the results of Theorem 1.1.

By Theorem 1.1, we see that all solutions of the corresponding homogeneous equation (7) of (8) are of $\sigma_{2}(f)=1$. By the variation of parameters, we see that all solutions of equation $(8)$ satisfy $\sigma_{2}(f) \leq 1$. If $\sigma_{2}(f)<1$, then $f$ clearly satisfies $\sigma_{e}(f)=0$, so $f$ is subnormal. Hence we see that all other solutions $f$ of equation (8) satisfy $\sigma_{2}(f)=1$ at most one subnormal solution.

Proof of Theorem 1.2. We divide into two cases:

Case 1. $A(z), B(z)$ are polynomials. Suppose that $p, q, m, n$ are constants,

$$
\begin{aligned}
& A(z)=A_{p} z^{p}+A_{p-1} z^{p-1}+\cdots+A_{0}, B(z)=B_{q} z^{q}+B_{q-1} z^{q-1}+\cdots+B_{0}, \\
& P(z)=a_{m} z^{m}+a_{m-1} z^{m-1}+\cdots+a_{0}, Q(z)=b_{n} z^{n}+b_{n-1} z^{n-1}+\cdots+b_{0} .
\end{aligned}
$$

Subcase 1.1. $m=n$.

(1) $p=q$. In this case, we give an example to illustrate that there exists nontrivial subnormal solution. The subnormal solution $f=e^{-z}$ satisfies the equation

$$
f^{\prime \prime}+\left(e^{z^{k}}+1\right) f^{\prime}+e^{z^{k}} f=0, \quad k \in \mathbb{N} .
$$

(2) $p>q$. If $f$ is a subnormal solution, from (9), then we get

$$
\left|P\left(e^{A}\right)\right| \leq\left|\frac{f^{\prime \prime}}{f^{\prime}}\right|+\left|Q\left(e^{B}\right)\right| \frac{f}{f^{\prime}} \mid .
$$

From Lemma 2.3, then there exists a set $E_{1} \subset(1,+\infty)$ with finite logarithmic measure and a constant $B>0$ such that for all $|z|=r \notin(0,1) \cup E_{1}$, we have

$$
\left|\frac{f^{\prime \prime}(z)}{f^{\prime}(z)}\right| \leq B(T(2 r, f))^{2} .
$$

From Lemma 2.4, there exists $r_{k} \notin(0,1) \cup E_{1}\left(r_{k} \rightarrow \infty\right)$, we get

$$
\left|\frac{f(z)}{f^{\prime}(z)}\right| \leq r_{k}^{2 s} \text {. }
$$

Take suitable $z=r_{k}$ or $z=-r_{k}$ to ensure that $A_{p} z^{p}>0$. From (10) and (13), we get

$$
\begin{aligned}
\left|a_{m}\right| e^{m\left|A_{p} r_{k}^{p}\right|}(1+o(1)) & \leq B\left(T\left(2 r_{k}, f\right)\right)^{2}+\left|b_{n}\right| e^{n\left|B_{q} r_{k}^{q}\right|} r_{k}^{2 s}(1+o(1)) \\
& \leq 2 B\left(T\left(2 r_{k}, f\right)\right)^{2}\left|b_{n}\right| e^{n\left|B_{q} r_{k}^{q}\right|} r_{k}^{2 s}(1+o(1))
\end{aligned}
$$

Since $m=n, p>q$, combining (12) with $0<\varepsilon<\frac{1}{12}$, then (14) changes into the following form

$$
\left|a_{m}\right| e^{m\left(\left|A_{p} r_{k}^{p}\right|-\left|B_{q} r_{k}^{q}\right|\right)}(1+o(1)) \leq 2 B e^{4 r_{k} \varepsilon} \cdot r_{k}^{2 s}<2 B e^{\frac{1}{3} r_{k}} r_{k}^{2 s} .
$$

Obviously, it is a contradiction. So, there is no subnormal solution in this case. 
(3) $p<q$. If $f$ is a subnormal solution, from Lemma (2.3), there exists a set $E_{2} \subset(1,+\infty)$ with finite logarithmic measure and a constant $B>0$ such that for all $|z|=r \notin(0,1) \cup E_{2}$, take either $z=r$ or $z=-r$ to assure that $B_{q} z^{q}>0$, we get

$$
\begin{aligned}
\left|Q\left(e^{B}\right)\right| & =\left|b_{n}\right| e^{n B_{q} r^{q}}(1+o(1)) \\
& \leq\left|\frac{f^{\prime \prime}}{f}\right|+\left|P\left(e^{A}\right)\right|\left|\frac{f^{\prime}}{f}\right| \\
& \leq B(T(2 r, f))^{3}+\left|a_{m}\right| e^{m\left|A_{p} r^{p}\right|} B(T(2 r, f))^{2}(1+o(1)) \\
& \leq 4 B(T(2 r, f))^{3}\left|a_{m}\right| e^{m \mid A_{p} r^{p}}(1+o(1)) .
\end{aligned}
$$

Using the similar argument as the proof of Theorem 1.1, we get a contradiction.

Subcase 1.2. $m<n$.

(1) $p=q$. If $f$ is a solution of equation (9), we assert that $\sigma_{2}(f)=p$, and $f$ is not a subnormal solution. Since Lemma 2.1, then we get

$$
\sigma_{2}(f) \leq \max \{\sigma(P(z)), \sigma(Q(z))\}=p .
$$

Using the similar proof of Theorem 1.1, we get the following inequality

$$
\left|b_{n}\right| e^{(n-m) r^{p}}(1+o(1)) \leq 4 B(T(2 r, f))^{3}\left|a_{m}\right|(1+o(1)) .
$$

Since $m<n$, then $(16)$ gives $\sigma_{2}(f) \geq p$. So $\sigma_{2}(f)=p$. If $f$ is a subnormal solution, combining (12) and (16), we get a contradiction.

(2) $p<q$. We can get a similar inequality to (16) as follows,

$$
\left|b_{n}\right| e^{n r^{q}-m r^{p}} \leq 4 B(T(2 r, f))^{3}\left|a_{m}\right|(1+o(1)) .
$$

If $f$ is a subnormal solution, combining (12) and (17), we also get a contradiction as $r \rightarrow \infty$.

(3) $p>q$. In this case, using the similar method as (2) of Subcase 1.1, we obtain that there is no subnormal solution of equation (9).

Subcase 1.3. $m>n$. Similar argument as Subcase 1.2, we get that there is no subnormal solution of equation (9).

Case 2. If either $A(z)$ or $B(z)$ is a transcendental entire function, without loss of generality, we assume that $A(z)$ is a transcendental entire function and $f$ is a solution of (9). From (9), Lemma 2.3 and Lemma 2.4, we get that there exists $E_{3} \subset(1,+\infty)$ with finite logarithmic measure and a constant $B>0$ such that for all $|z|=r_{k} \notin(0,1) \cup E_{3}$, we have

$$
\left|a_{m}\right| e^{m M\left(r_{k}, A(z)\right)} \leq 4 B\left(T\left(2 r_{k}, f\right)\right)^{3}\left|b_{n}\right| e^{n M\left(r_{k}, B(z)\right)} r_{k}^{2}(1+o(1)),
$$

where $M\left(r_{k}, f\right)=\max _{|z|=r_{k}}|f(z)|$.

Subcase 2.1. Suppose that $\sigma_{p}(A(z))>\sigma_{p}(B(z))$, thus from (18) and $A(z)$ is transcendental entire, we get $\sigma_{e}(f)=\infty$. Hence $f$ is not a subnormal solution. 
Subcase 2.2. Suppose that $\sigma_{p}(A(z))<\sigma_{p}(B(z))$. Similarly as above, we get $\sigma_{e}(f)=\infty$, hence $f$ is not a subnormal solution.

Subcase 2.3. Suppose that $\sigma_{p}(A(z))=\sigma_{p}(B(z))$ and $m=n$. We give the following example to illustrate there may exist a subnormal solution in this case. A subnormal solution $f=e^{-z}$ satisfies the equation

$$
f^{\prime \prime}+\left(\exp ^{[n+1]} z+1\right) f^{\prime}+\left(\exp ^{[n+1]} z\right) f=0 .
$$

Thus, we have completed the proof of Theorem 1.2.

Acknowledgments. The authors thank the referees for their careful reading of the paper and insightful comments.

\section{References}

[1] T. B. Cao and H. X. Yi, On the complex oscillation of higher order linear differential equations with meromorphic coefficients, J. Syst. Sci. Complex. 20 (2007), no. 1, 135148.

[2] Z. X. Chen and K. H. Shon, On subnormal solutions of second order linear periodic differential equations, Sci. China Ser. A 50 (2007), no. 6, 786-800.

[3] Y. M. Chiang and S. A. Gao, On a problem in complex oscillation theory of periodic second order linear differential equations and some related perturbation results, Ann. Acad. Sci. Fenn. Math. 27 (2002), no. 2, 273-290.

[4] G. Gundersen, Estimates for the logarithmic derivative of a meromorphic function, plus similar estimates, J. London Math. Soc. (2) 37 (1988), no. 1, 88-104.

[5] G. Gundersen and E. M. Steinbart, Subnormal solutions of second order linear differential equations with periodic coefficients, Results Math. 25 (1994), no. 3-4, 270-289.

[6] G. Jank and L. Volkmann, Einführung in die Theorie der ganzen und Meromorphen Funktionen mit Anwendungen auf Differentialgleichungen, Birkhäuser Verlag, Besel, 1985 .

[7] L. Kinnunen, Linear differential equations with solutions of finite iterated order, Southeast Asian Bull. Math. 22 (1998), no. 4, 385-405.

[8] I. Laine, Nevanlinna Theory and Complex Differential Equations, de Gruyter Studies in Mathematics, 15. Walter de Gruyter \& Co., Berlin, 1993.

[9] H. Wittich, Subnormale Losungen der Differentialgleichung: $w^{\prime \prime}+p\left(e^{z}\right) w^{\prime}+q\left(e^{z}\right) w=0$, Nagoya Math. J. 30 (1967), 29-37.

[10] C. C. Yang and H. X. Yi, Uniqueness Theory of Meromorphic Functions, Mathematics and its Applications, 557. Kluwer Academic Publishers Group, Dordrecht, 2003.

KAI LIU

SCHOOL OF MATHEMATiCS

SHANDONG UNIVERSITY

Jinan, Shandong, 250100, P. R. China

E-mail address: liukai418@126.com

LIAN-ZHONG YANG

SCHOOL OF MATHEMATiCS

SHANDONG UNIVERSITY

Jinan, Shandong, 250100, P. R. China

E-mail address: lzyang@sdu.edu.cn 